\title{
Análise da Produção Científica Brasileira sobre o Teste das Matrizes Progressivas de Raven
}

\author{
Lucila Moraes Cardoso \\ Universidade Estadual do Ceará, CE, Brasil. \\ Jamille Cavalcante de Oliveira \\ Hospital Universitário Walter Cantídio, CE, Brasil.
}

\author{
Érica Ive Xavier Lopes \\ Universidade Estadual do Ceará, CE, Brasil. \\ Aline Pinheiro Braga \\ Universidade Estadual do Ceará, CE, Brasil.
}

Resumo: OTeste das Matrizes Progressivas de Raven é um instrumento não verbal para avaliação da inteligência. As pesquisas sobre ele, no Brasil, iniciaram-se na década de 1950 e continuaram a desenvolver-se com o passar dos anos, em maior ou menor escala de acordo com o período. O presente estudo objetivou quantificar e analisar a produção científica brasileira de artigos a respeito do Teste das Matrizes Progressivas de Raven, disponíveis nas bases de dados Scientific Electronic Library Online (SciELO) e Periódicos Eletrônicos em Psicologia (PePSIC). Foram selecionados e analisados 71 artigos obtidos a partir das palavras-chaves "teste de Raven" e "Raven". As produções científicas analisadas foram publicadas no período entre 1995 e 2014 e, em sua maioria, eram provenientes da região Sudeste brasileira. Notou-se que a maioria dos artigos referiu o uso do teste de Raven em contextos específicos, e poucos buscaram analisar as qualidades psicométricas do instrumento, evidenciando a demanda de mais estudos sobre as qualidades psicométricas do teste de Raven.

Palavras-chave: Matrizes Progressivas de Raven, Pesquisa Bibliográfica, Avaliação Psicológica.

\section{Analysis of the Brazilian Scientific Production on the Raven's Progressive Matrices Test}

\begin{abstract}
The Raven's Progressive Matrices Test is a non-verbal tool for assessment of intelligence, whose research in Brazil started in the 1950s and continued over the years, to a greater or lesser extent according to the period. This study aimed to quantify and analyze the Brazilian scientific production of articles concerning the Raven's Progressive Matrices Test, available in the Scientific Electronic Library Online (SciELO) and Electronic Journal of Psychology (PePSIC) databases. Seventy-one articles using the keywords "Raven test" and "Raven" were selected and analyzed. The analyzed scientific works were published between 1995 and 2014 and mostly come from the Brazilian southeast region. It was noted that most of the articles reported the use of Raven Test in specific contexts and few sought to analyze the psychometric qualities of the instrument, evidencing the need for more studies on the psychometric qualities of the Raven Test.

Keywords: Raven Progressive Matrices, Bibliographical Research, Psychological Assessment.
\end{abstract}




\title{
Análisis de la Producción Científica Brasileña acerca de la Prueba de Raven
}

\begin{abstract}
Resumen: El Test de Matrices Progresivas de Raven es un instrumento no verbal para evaluación de la inteligencia, cuyas investigaciones en Brasil iniciaron en la década de 1950 y continuaron con el paso de los años, en mayor o menor medida de acuerdo con el período. El presente estudio tuvo como objetivo cuantificar y analizar la producción científica brasileña de artículos sobre el Test de Matrices Progresivas de Raven, disponibles en las bases de datos Scientific Electronic Library Online (SciELO) y Revistas Electrónicas en Psicología (PePSIC). 71 artículos, obtenidos a partir con las palabras clave "test de Raven" y "Raven" fueron seleccionados y analizados. Las producciones científicas analizadas fueron publicadas en el período entre 1995 y 2014 y provienen, en su mayoría, de la región sudeste brasileña. Se notó que la mayoría de los artículos relataron el uso del test de Raven en contextos específicos, y pocos buscaron analizar las cualidades psicométricas del instrumento, evidenciando la demanda por más estudios sobre las cualidades psicométricas del test de Raven.
\end{abstract}

Palabras clave: Matrices Progresivas de Raven, Investigación Bibliografica, Evaluación Psicológica.

\section{Introdução}

Atividade complexa e amplamente utilizada na Psicologia, a Avaliação Psicológica tem se constituído no Brasil como uma área de grande importância, principalmente após o reconhecimento da Psicologia como profissão e da delimitação de tal área como prática exclusiva dos psicólogos, por meio da Lei no 4.119 , de 1962 (Anache, \& Corrêa, 2010). A avaliação psicológica consiste em um processo de busca de conhecimentos a respeito do funcionamento psicológico das pessoas, podendo auxiliar na orientação das decisões e ações futuras. Para tanto, a área tem como uma de suas principais atividades o uso de instrumentos psicológicos, que têm por função a coleta de informações confiáveis que possam servir de base ao processo mais amplo da avaliação psicológica (Primi, 2010).

Para servir a este propósito, os testes psicológicos precisam ter suficiente embasamento científico e devem ser adequadamente utilizados. O Conselho Federal de Psicologia (CFP), em 2001, regulamentou pela primeira vez a elaboração, a comercialização e o uso dos testes psicológicos por meio da Resolução CFP n ${ }^{\circ}$ 25/2001 (CFP, 2001). Esta foi substituída pela Resolução CFP no 002/2003, que objetivou regulamentar os parâmetros mínimos necessários no que se refere às qualidades psicométricas dos instrumentos (CFP, 2003). Entre os requisitos técnicos necessários para um instrumento ser considerado adequado para uso estão a especificação do constructo que o instrumento visa avaliar, a fundamentação na literatura da área, a presença de evidências empíricas de validade, fidedignidade (também conhecida como precisão) e a apresentação de um sistema de correção e interpretação (Pacanaro, Alves, Rabelo, Leme, \& Ambiel, 2011).

As evidências de validade são importantes aspectos considerados na construção de um teste, pois se referem ao grau em que as interpretações obtidas dos dados empíricos do teste encontram sustentação em base científica sólida (Urbina, 2007). A fidedignidade é a consistência dos escores obtidos pelas mesmas pessoas em ocasiões diferentes. Além dessas duas qualidades psicométricas que foram sinteticamente definidas, há a padronização e normatização, que se referem, respectivamente, à uniformidade em todos os procedimentos relativos ao uso do teste e aos padrões de como se deve interpretar os escores obtidos nele (Pasquali, 2001).

Para assegurar esses princípios técnicos, no que se refere às qualidades dos instrumentos, o Conselho Federal de Psicologia criou o Sistema de Avaliação dos Testes Psicológicos (Satepsi), no final de 2001 (Primi, 2010). O Satepsi é composto por uma comissão consultiva de especialistas em avaliação psicológica, auxiliada por pareceristas ad hoc. Todos os membros da comissão e os pareceristas são pesquisadores e profissionais da área que recebem a indicação do CFP e voluntariamente têm como função analisar e dar parecer técnico sobre cada um dos instrumentos que é enviado para análise.

Ainda na perspectiva de divulgar e fortalecer a área, o ano de 2011 foi escolhido pelo CFP como o ano temático da Avaliação Psicológica, sendo promovidas atividades nas sedes e subsedes dos conselhos regionais e no 
próprio CFP, objetivando refletir sobre questões relativas aos temas (CFP, 2011). Embora sua importância já tenha sido devidamente reconhecida, a avaliação psicológica, assim como qualquer outra área de conhecimento, necessita de constantes avanços no que tange à qualidade dos estudos e das práticas desenvolvidas.

Dentre os instrumentos de avaliação psicológica, existe o Teste das Matrizes Progressivas de Raven (Raven Progressive Matrices - RPM). O instrumento foi criado por John C. Raven, na Escócia, sendo publicado e padronizado em 1938. O teste foi originalmente publicado como Matrizes Progressivas Standard e pretendia abarcar todas as faixas etárias de desenvolvimento intelectual. Em 1947, foram desenvolvidas mais duas versões pelo mesmo autor, denominadas de Matrizes Progressivas Coloridas e Matrizes Progressivas Avançadas. As Matrizes Progressivas Standard são conhecidas no Brasil como Escala Geral, indicada para sujeitos de 12 a 65 anos; as Matrizes Progressivas Coloridas são conhecidas como Escala Especial, destina-se às crianças na faixa de 5 a 11 anos, deficientes mentais e pessoas idosas e, por fim, as Matrizes Progressivas Avançadas, que são designadas para o uso com pessoas que tenham mais de 11 anos e inteligência superior à media, sendo mais utilizadas, portanto, para universitários (Angelini, Alves, Custódio, Duarte, \& Duarte, 1999).

As Matrizes Progressivas de Raven constituem testes não verbais para avaliação da inteligência, elaborados a partir do referencial da teoria bifatorial de Charles Spearman e que tem como objetivo avaliar a capacidade intelectual geral - fator " $g$ " de inteligência. As três escalas pretendem avaliar a capacidade edutiva do fator g, compreendida como a capacidade de extrair novos significados e informações de dados correlatos já conhecidos, sendo essa capacidade amplamente empregada na resolução de tarefas entre diversas áreas do conhecimento. Para melhor captar a origem do fator geral e, assim, evitar influências de questões culturais e de treinamento do aplicador, optou-se por itens de caráter gráfico em vez de verbal para o teste, seguindo, nesta elaboração, os princípios da teoria da Gestalt. Todos os itens do teste foram, portanto, elaborados com o intuito de que a solução ocorra dentro da percepção, espacial ou lógica, de uma configuração (gestalt) (Bandeira, Alves, Giacomel, \& Lorenzatto, 2004; Pasquali, Wechsler, \& Bensusan, 2002). Sendo assim, o conjunto de tarefas não verbais que compõem as RPM tem por finalidade medir capacidade do sujeito para apreender relações entre figuras.
As primeiras pesquisas com o Teste de Raven no Brasil iniciaram-se na década de 1960 (Bandeira et al., 2004) e continuaram a ser desenvolvidas com o passar das décadas, em maior ou menor escala de acordo com o período. Nesse sentido, torna-se relevante compreender como têm sido conduzidos os estudos brasileiros que utilizam as RPM. Segundo Witter (1999), a análise das produções científicas permite visualizar mudanças no fluxo destas produções, como também apontar áreas que já foram muito pesquisadas e áreas que ainda demandam novas pesquisas.

Três importantes estudos, que buscaram analisar a produção científica brasileira na área da avaliação psicológica, foram considerados relevantes nesta pesquisa, a saber, o estudo de Joly, Berberian, Andrade e Teixeira (2010), de Joly, Silva, Nunes e Souza (2007) e de Souza Filho, Belo e Gouveia (2006). Na pesquisa feita por Joly et al. (2010), foi analisada a produção científica de teses e dissertações em avaliação psicológica no Brasil. Dentre os resultados encontrados, pôde-se constatar que mais da metade dos resumos eram de dissertações de mestrado, sendo a região Sudeste responsável pela maior parte da produção científica de avaliação psicológica. Verificou-se também que a maioria dos estudos tinha por objetivo buscar parâmetros psicométricos, sendo os construtos mais estudados a personalidade e a inteligência.

A pesquisa feita por Joly et al. (2007) analisou os resumos de painéis dos três Congressos Nacionais de Avaliação Psicológica, e descobriu que a produção foi predominantemente feminina, com autores filiados a instituições públicas e particulares de ensino, procedentes da região Sudeste do Brasil. Foi encontrada uma grande variedade de testes psicológicos citados, em sua maioria testes objetivos, de inteligência e personalidade. Já o estudo de Souza Filho et al. (2006) buscou traçar o perfil da utilização dos testes psicológicos na literatura científica brasileira entre os anos de 2000 e 2004 e constatou que a maior concentração dessas produções estava situada na Região Sudeste, sendo as universidades públicas as mais produtivas nessa modalidade. Esse estudo concluiu que a utilização dos testes psicológicos no contexto da produção científica nacional ainda era modesta e estava, em sua maioria, restrita aos âmbitos acadêmicos mais intensamente dedicados ao estudo dos instrumentos psicológicos.

É possível afirmar, portanto, que estudos de revisão de literatura permitem focalizar determinados aspectos da produção que se busca conhecer, além de 
tornar possível a análise da qualidade e da efetividade do conhecimento que está sendo produzido. Tendo em vista o que foi exposto, o presente estudo teve por objetivo analisar a produção científica brasileira de artigos a respeito do Teste das Matrizes Progressivas de Raven, traçando assim um panorama dos estudos e da utilização do testes retratados nas pesquisas encontradas.

\section{Método}

\section{Procedimentos}

Os artigos foram obtidos por meio de uma busca na Biblioteca Virtual de Saúde - Psicologia Brasil (BVS-Psi Brasil), consultando as bases de dados completas Scientific Eletronic Library Online (SciELO Brasil) e Periódicos Eletrônicos em Psicologia (PePSIC). Foram selecionados os artigos publicados entre 1995 e 2014, que utilizaram o teste em suas pesquisas e cujos estudos foram desenvolvidos no Brasil. Os critérios de exclusão estabelecidos foram, portanto, estudos realizados em países estrangeiros, que não utilizassem de fato o teste de Raven no decorrer da pesquisa ou cujos dados dos autores não pudessem ser encontrados.

Primeiramente, foi realizada a busca pelo termo “Teste de Raven", em que foram encontrados 16 artigos na base de dados PePSIC e 41 na base de dados SciELO. Em seguida, foi realizada uma busca pelo termo "Raven" no BVS-Psi, em que foram disponibilizados 25 artigos na base de dados PePSIC e 68 na base de dados SciELO. Do total de 150 artigos, 49 apareceram duas vezes nas buscas, quatro apareceram quatro vezes e 36 apareceram uma única vez, totalizando a quantidade de 89 artigos. Destes, 18 não foram incluídos por apresentarem algum dos critérios de exclusão já citados. Sendo assim, foram considerados para esta revisão de literatura 71 trabalhos completos.

Os 71 artigos obtidos foram lidos e quantificados conforme algumas categorias pré-determinadas de análise. As categorias estabelecidas foram: (1) Autoria, com dados sobre a quantidade de autores por artigo e informações sobre o sexo, a titulação, a afiliação institucional e a graduação do primeiro autor; (2) Instituição, na qual considerou-se o tipo de Unidade administrativa da Instituição de vínculo do primeiro autor, bem como a Unidade da Federação das instituições; e (3) os dados pesquisa em si, incluindo, objetivo, participantes e o modelo do teste de Raven utilizado.

\section{Resultados e discussão}

Os dados foram organizados em uma planilha Excel e, em seguida, foram analisadas as frequências de cada uma das categorias. No Quadro constam as informações de autoria, periódico em que foram publicados os estudos e escalas do Raven utilizadas nos 71 artigos considerados às análises.

Totalizaram-se 32 periódicos responsáveis pela publicação dos artigos encontrados. Dentre eles, os que apresentaram a maior quantidade de publicações foram as revistas Psicologia - Reflexão e Crítica, com 12 publicações (16,9\%), Estudos de Psicologia (Campinas), com seis publicações $(8,45 \%)$, Psicologia - Teoria e Pesquisa, também com seis publicações (8,45\%) e Avaliação Psicológica, com cinco publicações $(7,04 \%)$. Essas revistas foram classificadas, respectivamente, nos estratos A1, A2, A1 e B1 do Qualis Web para Psicologia, três delas possuindo a classificação mais elevada na estratificação da qualidade da produção intelectual proposta pela Qualis. Tal fato aponta para o espaço e reconhecimento que boa parte das pesquisas encontradas alcançaram no meio científico, além da qualidade e seriedade com que tais estudos foram conduzidos, já que vários são os critérios e exigências para a publicação de artigos nos periódicos em questão.

Na Tabela 1 é possível identificar o ano em que os artigos foram publicados. Nota-se que os primeiros trabalhos completos com o Raven disponibilizados por meio eletrônico datam da década de 1990, em que três publicações foram encontradas. De acordo com Primi (2010), foi a partir desta década que houve uma maior movimentação na área por meio da organização de eventos dedicados à avaliação psicológica.

Nota-se que, a partir de 2004, houve um aumento no número de pesquisas utilizando as RPM. Tal fato pode ter associação com a resolução CFP $\mathrm{n}^{\circ} 02 / 2003$, conforme foi também discutido por Joly et al. (2010), ao fazer análise das teses e dissertações em Avaliação Psicológica disponíveis na BVS-PSI Brasil. Além disso, pode ser observado um pico na frequência de publicações no ano de 2012 (16,9\% dos artigos encontrados). Esse aspecto pode ter relação com o Ano Temático da Avaliação Psicológica, que contou com seminários em âmbito regional e nacional entre os anos de 2011 e 2012, sugerindo que as ações no sentido de divulgação e debate sobre a área tenham sido produtivas (CFP, 2011).

$\mathrm{Na}$ análise da frequência de autores por artigos, conforme Tabela 2 , observa-se que $56(78,87 \%)$ foram produzidos por três ou mais autores. O número de 
Quadro

Estudos sobre o teste de Raven constantes nas bases de dado SciELO e PePSIC.

\begin{tabular}{l} 
Autores \\
\hline Wigg, \& Duro (1995) \\
Capovilla, Capovilla, Macedo, Costa, \& Duduchi (1996) \\
Alves (1998) \\
Linhares, Carvalho, Bordin, Chhimello, Martinez, \& Jorge \\
(2000) \\
Carvalho, Linhares, \& Martinez (2001) \\
Bordin, Linhares, \& Jorge (2001) \\
Ferriolli, Linhares, Loureiro, \& Marturano (2001) \\
Pasquali, Wechsler, \& Bensusan (2002) \\
Santos et al. (2002)
\end{tabular}

Fonseca, Tedrus, Martins, Gibert, Antunes, \& Laloni (2003)

Santos, Raspantini, Silva, \& Escrivão (2003)

Bandeira et al. (2004)

Dell'Aglio, \& Hutz (2004)

Dias, Enumo, \& Azevedo Júnior (2004)

Leon, Bosa, Hugo, \& Hutz (2004)

Macedo, Andreucci, \& Montelli (2004)

Okano, Loureiro, Linhares, \& Marturano (2004)

Barbosa, Simonetti, \& Rangel (2005)

Linhares, Chimello, Bordin, Carvalho, \& Martinez (2005)

Dias, \& Enumo (2006)

Gera, \& Linhares (2006)

Rodrigues, Caminha, \& Horta (2006)

Marín Rueda, \& Sisto (2006)

Sisto, Marin Rueda, \& Bartholomeu (2006)

Flores-Mendoza, Mansur-Alves, Lelé, \& Bandeira (2007)

Flores-Mendoza, \& Nascimento (2007)

Fonseca, Tedrus, Pacheco, Berretta, Campregher, \& Costa (2007)

Lukasova, Macedo, Valois, Macedo, \& Schwartzman (2007)

Paula, \& Enumo (2007)

Bandeira, Costa, \& Arteche (2008)

Ogusuko, Lukasova, \& Macedo (2008)

Pawlowski, Fonseca, Salles, Parente, \& Bandeira (2008)

Guilhoto, Fernandes, Pacheco, Ballester, \& Gilio (2009)

Maia, Soares, \&Victoria (2009)

Mian, Tango, Lopes, \& Loureiro (2009)

Pereira, Santos, \& Williams (2009)

Rosseti, Rabelo, Leme, Pacanaro, \& Güntert (2009)

Flores-Mendoza, Mansur-Alves, Abad, \& Lelé (2010)

Flores-Mendoza, Jardim, Abad, \& Rodrigues (2010)

Periódico

Raven

Psicologia USP

Especial e geral

Psicologia Escolar e Educacional

Avançada

Paidéia (Ribeirão Preto)

Especial

Psicologia: Reflexão e Crítica

Especial

Psicologia: Teoria e Pesquisa

Especial e geral

Psicologia: Reflexão e Crítica

Especial

Avaliação Psicológica

Especial

Caderno de Saúde Pública

Especial

Geral

Arquivos de Neuro-psiquiatria

Especial

PSIC - Rev. de Psic. da Vetor Ed.

Especial

Psicologia em Estudo

Especial

Psicologia: Reflexão e Crítica

Especial

Psicologia em Estudo

Especial

Avaliação Psicológica

Especial

Arquivos de Neuro-psiquiatria

Especial

Psicologia: Reflexão e Crítica

Especial

Rev. Bras. de Educação Especial

Geral

Psicologia: Reflexão e Crítica

Especial

Psicologia: Teoria e Pesquisa

Especial

Psicologia: Reflexão e Crítica

Especial

Rev. Bras. de Terapias Cognitivas

Aletheia

Geral

Psicologia: Reflexão e Crítica

Especial

Psicologia: Reflexão e Crítica

Especial

Especial

Estudos de Psicologia (Campinas) Especial

Arquivos de Neuro-psiquiatria

Especial

Psico-USF

Geral

Rev. Bras. de Educação Especial

Especial

Psicologia: Reflexão e Crítica

Especial

Psicologia Teoria e Prática

Geral

Arquivos Brasileiros de Psicologia Geral

Journal of Epilepsy and Clinical

Neurophysiology

Especial e geral

Estudos e Pesquisas em Psicologia Geral

Psicologia: Teoria e Pesquisa

Especial

Psicologia: Teoria e Pesquisa

Especial

Psico-USF

Avançada

Boletim Psicologia

Especial

Estudos de Psicologia (Campinas) Especial e geral

Avaliação Psicológica

Especial

Continua 
Continuação

Paulino, \& Lopes (2010)

Ferreira, Conte, \& Marturano (2011)

Ribeiro, Semer, \& Yazigi (2011)

Silva, \& Santos (2011)

Varanda, \& Fernandes (2011)

Busnello, Jou, \& Sperb (2012)

Flores-Mendoza, Widaman, Mansur-Alves, Silva Filho, Pasian, \& Schlottfeldt (2012)

Ikeda, Horta, Bruscato, \& Dolci (2012)

Lobato, Miranda, Faria, Bethony, \& Gazzinelli (2012)

Nunes et al. (2012)

Osborn, \& Pereira (2012)

Santos, \& Nascimento (2012)

Silva, Zaninotto, Lucia, \& Scaff (2012)

Silva, Assis, Hasselmann, Santos, Pinto, \& Rodrigues (2012)

DaSilveira, \& Gomes (2012)

Souza, \& Resende (2012)

Zampieri, Schelini, \& Crespo (2012)

Cardoso, Silva, \& Pereira (2013)

Ferreira, Ferreira, Lima, \& Cruz (2013)

Fonseca, Albernazb, Kaufmann, Neves, \& Figueiredo (2013)

Mansur-Alves, Flores-Mendoza, \& Tierra-Criollo (2013)

Novaes, Mishima, \& Santos (2013)

Queiroz, Enumo, \& Primi (2013)

Soares, Francischetto, Peçanha, Miranda, \& Dutra (2013)

Costa et al. (2014)

Czermainski, Riesgo, Guimarães, Salles, \& Bosa (2014)

Flores-Mendoza, Widaman, Bacelar, \& Lelé (2014)

Murphy, Zachi, Roque, Ventura, \& Schochat (2014)

Saur, Correia, Bettiol, Barbieri, \& Loureiro (2014)

Werlang, Nunes, \& Borges (2014)
Psicologia: Ciência e Profissão

Estudos de Psicologia (Campinas)

Psicologia: Reflexão e Crítica

Psicologia: Teoria e Pesquisa

Jornal da Sociedade Brasileira de

Fonoaudiologia

Psicologia: Reflexão e Crítica

Temas em Psicologia

Brazilian Journal of

Othorinolaryngology

Revista da Sociedade Brasileira de Medicina Tropical

Avaliação Psicológica

Einstein

Estudos de Psicologia (Campinas)

Psicologia Hospitalar

Jornal de Pediatria

Paidéia (Ribeirão Preto)

Avaliação Psicológica

Estudos de Psicologia (Campinas)

CoDAS

Jornal Brasileiro de Psiquiatria

Jornal de Pediatria

Psicologia: Reflexão e Crítica

Revista de Psicopedagogia

Revista Brasileira de Educação

Especial

Estudos de Psicologia (Campinas)

Revista Brasileira de Psiquiatria

Paidéia (Ribeirão Preto)

Arquivos Brasileiros de Psicologia

CoDAS

Psico-USF

Psico-USF
Avançada

Especial

Especial

Especial

Especial

Especial

Geral e

avançada

Especial

Especial

Avançada

Especial

Geral

Especial

Especial e geral

Avançada

Geral

Especial

Especial

Especial e geral

Especial

Especial

Especial

Especial

Geral

Especial

Especial

Geral

Especial e geral

Especial

Especial autores por artigo mostra o acentuado predomínio de publicações em sistema de coautoria, sugerindo que o instrumento tem sido utilizado por grupos de pesquisas. Informações semelhantes foram obtidas por Souza Filho et al. (2006), em sua análise da produção científica brasileira acerca dos testes psicológicos. Este afirma, ainda, ser o sistema de coautoria um elemento facilitador que viabiliza a produção e minimiza as dificuldades nas pesquisas no campo dos testes.

Outro dado que chamou atenção sobre a autoria dos artigos foi o fato de $19(26,76 \%)$ terem como primeiros autores profissionais não psicólogos, tais como médicos, enfermeiros, fonoaudiólogos, dentre outros. Desses 19 trabalhos, 12 (63,16\%) possuíam pelo menos um psicólogo na equipe de pesquisa e, dos sete estudos sem psicólogo explicitamente envolvido na equipe, cinco foram desenvolvidos por autores com mestrado, doutorado ou pós-doutorado em Psicologia. Por serem os testes psicológicos instrumentos de uso exclusivo do psicólogo, como dispõe o artigo 13 da Lei no 4.119, de 1962 (Anache, \& Corrêa, 2010), pesquisas que envolvam tais instrumentos devem ter 
em sua equipe algum profissional da área. Este dado aponta, portanto, para a necessidade de um maior cuidado quanto ao uso de testes psicológicos em pesquisas, já que este uso deve ser feito pelos profissionais que de fato possuem o conhecimento e a competência para tal.

A predominância de autores do sexo feminino nas pesquisas encontradas é marcante e significativa, já que $81,69 \%$ dos primeiros autores dos artigos encontrados são mulheres e, dentre os que possuem formação em Psicologia, este índice aumentou para $84,62 \%$. O dado reflete uma tendência geral da Psicologia brasileira, que é a de possuir, em seus quadros, uma predominância do sexo feminino (Souza Filho et al., 2006; Joly et al., 2007; 2010).

Outro dado que corrobora aqueles observados na produção científica na área da Psicologia é a concentração de instituições proponentes de pesquisas localizadas na região Sudeste. Esse dado pode ser verificado na Tabela 3, em que são fornecidas informações sobre a instituição de origem do primeiro autor de cada artigo, bem como o estado e a região brasileira onde se localizam tais instituições. Apesar de existir

Tabela 1

Frequência de artigos publicados por ano.

\begin{tabular}{lcc}
\hline Ano de publicação & Frequência & $\%$ \\
\hline 1995 & 1 & 1,41 \\
1996 & 1 & 1,41 \\
1998 & 1 & 1,41 \\
2000 & 1 & 1,41 \\
2001 & 3 & 4,22 \\
2002 & 2 & 2,82 \\
2003 & 2 & 2,82 \\
2004 & 6 & 8,45 \\
2005 & 2 & 2,82 \\
2006 & 5 & 7,04 \\
2007 & 5 & 7,04 \\
2008 & 3 & 4,22 \\
2009 & 5 & 7,04 \\
2010 & 4 & 5,64 \\
2011 & 5 & 7,04 \\
2012 & 12 & 16,9 \\
2013 & 7 & 9,86 \\
2014 & 6 & 8,45 \\
Total & 71 & 100 \\
\hline
\end{tabular}

a possibilidade de um mesmo estudo ter sido desenvolvido, simultaneamente, por diferentes autores em estados distintos, ainda assim destaca-se a importância deste dado, pois permite um vislumbre significativo dos locais onde majoritariamente estão sendo desenvolvidos estudos sobre o tema.

No que se refere às instituições de ensino superior, foram publicados artigos em 28 instituições, 12 delas particulares e 16 públicas. Autores afiliados às 16 universidades públicas foram responsáveis pela produção de $55(77,46 \%)$ estudos, os afiliados às 12 particulares foram responsáveis por $16(22,54 \%)$ estudos, como mostra a Tabela 4. No Brasil, tradicionalmente as universidades públicas permanecem à frente no âmbito da pesquisa e da produtividade científica. Ainda assim, nota-se que a porcentagem de instituições particulares também é significativa, indicando que estas instituições, que têm um histórico pautado prioritariamente no ensino, têm aumentado sua participação nas pesquisas, como constata Souza Filho, Belo e Gouveia (2006) e Joly et al. (2010).

Entre as instituições públicas, as que se destacaram no uso do teste de Raven em pesquisas foram a Universidade de São Paulo (22,53\%), a Universidade Federal de Minas Gerais $(14,08 \%)$ e a Universidade Federal do Rio Grande do Sul (11,26\%), totalizando

Tabela 2

Informações sobre a autoria dos artigos envolvendo o Teste de Raven.

\begin{tabular}{|c|c|c|c|}
\hline Categorias & Classificação & Frequência & $\%$ \\
\hline \multirow{3}{*}{$\begin{array}{l}\text { Quantidade } \\
\text { de autores }\end{array}$} & Um & 1 & 1,41 \\
\hline & Dois & 14 & 19,72 \\
\hline & Três ou mais & 56 & 78,87 \\
\hline \multirow{4}{*}{$\begin{array}{l}\text { Titulação } \\
\text { do primeiro } \\
\text { autor }\end{array}$} & Graduados & 13 & 18,31 \\
\hline & Mestrado & 21 & 29,58 \\
\hline & Doutorado & 27 & 38,03 \\
\hline & $\begin{array}{c}\text { Pós- } \\
\text { doutorado }\end{array}$ & 10 & 14,08 \\
\hline \multirow{2}{*}{$\begin{array}{l}\text { Primeiro } \\
\text { autor } \\
\text { psicólogo }\end{array}$} & Sim & 52 & 73,24 \\
\hline & Não & 19 & 26,76 \\
\hline \multirow{2}{*}{$\begin{array}{l}\text { Sexo do } \\
\text { primeiro } \\
\text { autor }\end{array}$} & Feminino & 58 & 81,69 \\
\hline & Masculino & 13 & 18,31 \\
\hline
\end{tabular}


Tabela 3

Informações sobre a instituição de origem dos primeiros autores dos artigos.

\begin{tabular}{lllcc}
\hline Categorias & Classificação & & Frequência & $\%$ \\
\hline Unidade & Pública & & 55 & 77,46 \\
administrativa & Particular & & 16 & 22,54 \\
& Nordeste & BA & 2 & 2,82 \\
& & DF & 2 & 2,82 \\
& Centro-oeste & GO & 1 & 1,41 \\
& & MT & 3 & 4,22 \\
Região/Estado & SP & MG & 11 & 43,66 \\
& Sudeste & RJ & 6 & 15,49 \\
& & ES & 3 & 8,45 \\
& & RS & 11 & 4,22 \\
& Sul & SC & 1 & 15,49 \\
& & & & 1,41 \\
\hline
\end{tabular}

47,87\% das publicações. Dentre as particulares, a que possui maior número de artigos envolvendo o teste de Raven é a Universidade São Francisco - Campus Itatiba $(4,22 \%)$.

A região Sudeste foi responsável pela produção de 51 artigos $(71,83 \%)$, mais da metade da produção encontrada, ressaltando-se o estado de São Paulo, responsável por $31(43,66 \%)$ das publicações. A região Nordeste produziu dois artigos, ambos advindos do estado da Bahia, e a região Norte não apresentou nenhuma produção. A concentração da produção de artigos que utilizaram o teste das Matrizes Progressivas de Raven em sua pesquisa na região Sudeste não é casual. Reflete a maior concentração de recursos disponíveis e maior volume de pesquisadores estabelecidos nessa região, como afirmam Souza Filho et al. (2006). Resultados similares foram encontrados nos estudos de Joly et al. (2007; 2010), que apontaram uma maior concentração da produção científica de avaliação psicológica também na região Sudeste.

A escassez de estudos a respeito do teste de Raven nas regiões Norte e Nordeste é um dado importante de ser avaliado, já que o Brasil, por ser um país de grande extensão territorial, possui peculiaridades regionais que precisam ser consideradas nos estudos sobre as qualidades psicométricas dos testes psicológicos. Como dispõe a resolução CFP n ${ }^{\circ}$ 02/2003, existem critérios mínimos que os instrumentos devem apresentar para serem considerados adequados para uso e comercialização (CFP, 2003), e tais critérios precisam ser analisados também em consonância com as particularidades de locais com realidades sociais, culturais e econômicas distintas.

No que se refere aos participantes das pesquisas, $76,05 \%$ foram crianças, 18,31\% adolescentes, $14,08 \%$ adultos, 9,86\% universitários e 1,41\% pacientes com TOC (Transtorno Obsessivo Compulsivo), como mostra a Tabela 4. Esse dado tem relação direta com o tipo de escala das Matrizes Progressivas de Raven utilizada nas pesquisas, também mostradas na Tabela 4 , pois em 76,05\% dos artigos foram utilizadas as Matrizes Progressivas Coloridas de Raven Escala Especial. Essa versão é a indicada para ser empregada com crianças com idade entre 5 e 11 anos, idosos ou pessoas que tenham alguma deficiência mental (Angelini et al., 1999). Esses dados sugerem a predominância de pesquisas realizadas com crianças e observa-se que essa tendência ocorre desde o começo da produção científica de artigos a respeito das RPM. Além disso, é importante ressaltar que oito artigos utilizaram em seus estudos duas versões distintas do Raven.

Ao fazer uma análise sobre os dados das pesquisas em si, como é mostrado na Tabela 4, observou-se que $55(77,46 \%)$ referiam-se a aplicação das RPM em contexto específico, 13 (18,31\%) analisavam as qualidades psicométricas de instrumentos, duas $(2,82 \%)$ utilizavam o teste enquanto critério de inclusão de sujeitos nas pesquisas desenvolvidas e uma $(1,41 \%)$ 
Tabela 4

Informações sobre as pesquisas.

\begin{tabular}{llcc}
\hline Categoria & & Frequência & $\%$ \\
\hline \multirow{5}{*}{ Participantes } & 54 & 76,05 \\
& Crianças & 13 & 18,31 \\
& Adolescentes & 10 & 14,08 \\
& Udultos & 7 & 9,86 \\
& Pacientes com TOC & 1 & 1,41 \\
& Matrizes Coloridas de Raven - Escala especial & 54 & 76,05 \\
\multirow{3}{*}{ Oodelo RPM } & Matrizes Progressivas de Raven - Escala geral & 19 & 26,76 \\
& Matrizes Avançadas de Raven - Escala avançada & 6 & 8,45 \\
& Aplicação das RPM em contexto específico & 55 & 77,46 \\
& Critério de Inclusão em Pesquisa & 2 & 2,82 \\
& Revisão de Literatura & 1 & 1,41 \\
& Evidências de Validade (RPM) & 5 & 7,04 \\
& Evidências de Validade (outros testes) & 7 & 9,86 \\
& Normatização & 1 & 1,41 \\
\hline
\end{tabular}

fazia revisão de literatura. Das pesquisas que buscaram analisar as qualidades psicométricas, 12 buscaram por evidências de validade, sendo cinco $(7,04 \%)$ especificamente sobre as RPM e o restante sobre outros instrumentos psicológicos, e um $(1,41 \%)$ referia-se à normatização das RPM. É importante ressaltar que, na revisão aqui desenvolvida, acessou-se apenas os estudos publicados em periódicos online.

Portanto, seis estudos pesquisaram sobre as qualidades psicométricas das RPM, em suas diferentes versões. O estudo de Pasquali et al. (2002) consistiu em estabelecer a estrutura interna do Raven, bem como seus parâmetros de validade e precisão para o Brasil, baseando-se numa amostra de 9.929 estudantes de escola pública do Distrito Federal. A idade dos participantes variou entre 5 e 11 anos e foi adotada a versão do Teste Raven Escala Especial na versão da Editorial Paidós. Por meio da análise fatorial, verificou-se que o teste de Raven Infantil é um teste adequado para avaliar o aspecto de raciocínio analógico de crianças brasileiras, ainda que alguns itens tenham sido considerados inconsistentes e outros irrelevantes.

O estudo de Sisto et al. (2006) teve como objetivo avaliar o ajuste do modelo Rasch quanto à unidimensionalidade das Matrizes Progressivas Coloridas de Raven. O teste foi aplicado numa amostra de 441 crianças, de primeira à quarta série do ensino fundamental, de uma escola municipal do estado de São Paulo. Os resultados obtidos sugeriram que a unidimensionalidade desta versão do Raven carece de sustentação e a pontuação total pode não ser uma medida suficiente para avaliar as habilidades envolvidas na resolução da tarefa proposta.

A pesquisa de Rosseti et al. (2009) teve como objetivo buscar evidências de validade das Matrizes Progressivas Avançadas de Raven em universitários no estado de São Paulo. O instrumento foi aplicado numa amostra de 369 estudantes dos cursos de Psicologia, administração de empresas, gestão de recursos humanos e pedagogia de ensino superior de duas universidades privadas. Os resultados apontaram para diferenças estatisticamente significativas na comparação entre homens e mulheres e entre os diferentes cursos. Ainda que tenha ocorrido uma diferença de acordo com a idade, esta não significativa do ponto de vista estatístico. Os autores do estudo, concluíram que o teste mostrou-se preciso para avaliar aspectos da inteligência geral em universitários.

No estudo de Nunes et al. (2012) buscou-se por evidências de validade das Matrizes Progressivas Avançadas de Raven por meio da correlação com a prova de Raciocínio Abstrato da Bateria de Provas de Raciocínio (BPR-5). Participaram do estudo 162 estudantes de uma universidade pública de Santa Catarina. 
Embora não tenha sido apontado como um objetivo da pesquisa, para assegurar a qualidade dos dados que seriam correlacionados, os autores também analisaram a consistência das respostas e observaram um nível de precisão excelente para ambas as medidas. Na análise da associação entre os instrumentos, observou-se correlação moderada no desempenho dos dois instrumentos. Porém, a média de dificuldade dos itens da Escala Avançada do Raven foi menor que a dos itens da prova de Raciocínio Abstrato da BPR-5, sugerindo que a Escala Avançada não seja uma medida que objetive avaliar exclusivamente pessoas com nível alto de inteligência, como também é possível que a prova Raciocínio Abstrato da BPR-5 não seja adequada para a população geral, mas sim para uma faixa da população com inteligência média-superior ou alta.

No estudo de Flores-Mendoza et al. (2014) foram investigados os parâmetros psicométricos das Matrizes Progressivas de Raven - Escala Geral para o estado de Minas Gerais. Para tal, utilizaram 1.956 protocolos do Raven coletados para situação de pesquisa com pessoas que tinham entre 7 e 65 anos. Na análise dos resultados, verificou-se que $13,8 \%$ dos itens do Raven foram considerados difíceis e $53,4 \%$ foram fáceis, divergindo da estimava de que a porcentagem entre itens classificados como difíceis e fáceis fosse entorno de $30,0 \%$ cada. Deste modo, os resultados sugerem que a amostra estudada teve facilidade para responder o teste Raven. Os autores sugeriram que essa facilidade pode ser compreendida pelo fenômeno conhecido como efeito "Flynn", segundo o qual há ganhos cognitivos ao longo das gerações.

A pesquisa desenvolvida por Bandeira et al. (2004) objetivou estabelecer normas às crianças de Porto Alegre, no Rio Grande do Sul. A amostra foi composta por 779 crianças, de 4 anos e 9 meses a 11 anos e 9 meses, matriculadas na rede pública estadual. Dentre os resultados, foi constatado aumento progressivo nas médias de acordo com a idade e não foram encontradas diferenças entre meninos e meninas. Além disso, foi feita uma comparação com as crianças de escolas públicas de São Paulo e as médias das crianças de Porto Alegre obtiveram médias mais altas. Reforçou-se, portanto, a necessidade do estabelecimento de normas distintas do Raven para as diferentes regiões do Brasil.

A escala especial do Raven foi utilizada em dois dos estudos sobre as qualidades psicométricas, sendo que um indicou bons resultados de precisão ainda que alguns itens tenham sido considerados inconsistentes
(Pasquali et al., 2002) e o outro apontou a demanda de novos estudos para sustentar o pressuposto unidimensionalidade do teste (Sisto et.al, 2006). A escala avançada também foi adotada em dois estudos com universitários, sendo que um deles buscou evidências de validade e concluiu que o teste era preciso (Rosseti et al., 2009) e o outro buscou por evidências de validade (Nunes et al., 2012), sugerindo que a escala avançada talvez não fosse indicada para uso exclusivo de pessoas com nível alto de inteligência. Por fim, um único estudo usou a escala geral (Flores-Mendoza et al., 2014), no entanto a descrição da amostra não diferenciou a quantidade de crianças, adultos e idosos e não há especificação sobre o grau de escolaridade da amostra, dificultando que se possa verificar se a escala geral de fato seria a versão mais indicada para faixa etária e escolaridade dos participantes.

A escassez de estudo que visam estudar as qualidades psicométricas do Raven dificulta que numa revisão de literatura se possa ter uma compreensão mais específica sobre a qualidade dos instrumentos. Assim, corrobora-se com a defesa de Pacanaro et al. (2011), Nunes et al. (2012) e Flores-Mendoza et al. (2014), entre outros, sobre a necessidade de pesquisas que busquem assegurar as qualidades psicométricas dos instrumentos. Os resultados claramente indicaram que a quantidade de estudos com tal objetivo é parco quando comparado à totalidade de artigos que utilizaram o instrumento pressupondo que se trata de um teste adequado para avaliação da inteligência. Destaca-se, deste modo, a relevância de novas estudos com o Raven para a preservação da qualidade e da confiabilidade do teste, minimizando os riscos de uso, correção e interpretação indevidos (Pasquali, 2001; Urbina, 2007).

\section{Considerações finais}

O levantamento dos artigos nas bases de dados SciELO e PePSIC possibilitou a visualização de como as pesquisas científicas em torno do teste de Raven têm sido realizadas. Os resultados obtidos mostraram a predominância de pesquisas que visavam o uso das RPM para situações de avaliação, sendo realizadas poucas pesquisas que estudassem as qualidades psicométricas do teste. Pesquisas que busquem, continuamente, atestar as qualidades dos instrumentos psicológicos são de essencial importância na área da avaliação psicológica, já que é por meio destas que o uso dos testes torna-se uma prática confiável. 
É importante, portanto, que a escassez de publicação ora apontada seja um dado que, ao mesmo tempo, alerte e instigue os pesquisadores da área.

Outro ponto importante diz respeito à concentração da maior parte dos trabalhos encontrados nas regiões Sul e Sudeste. A pouca participação da região Nordeste, Centro-Oeste e a ausência de artigos advindos da região Norte são dados que refletem uma realidade que persiste no Brasil e que necessita ser transformada, pois ainda é o eixo Sul-Sudeste que concentra maior volume de pesquisadores e de recursos disponíveis para a realização destes estudos. São grandes as diferenças culturais, socioeconômicas e históricas entre as diversas regiões do país, sendo assim de fundamental importância que estudos envolvendo o instrumento possam desenvolver-se também nas regiões que apresentam atualmente menor produção científica acerca da temática.

É interessante ressaltar, no entanto, que a revisão de literatura aqui desenvolvida é parcial, já que se baseou nos artigos encontrados em apenas duas bases de dados, não levando em consideração os estudos publicados em revistas que não estão online e as pesquisas psicométricas presentes nos próprios manuais das escalas Colorida e Standard do Raven. Apesar disso, esta pesquisa permitiu a visualização das principais nuances e transformações que perpassaram a trajetória das produções científicas acerca do teste de Raven ao longo dos anos no Brasil. Seria importante que estudos como esse também levassem em conta a análise das produções científicas utilizadas em outros países para que fosse possível desenvolver em uma perspectiva internacional a trajetória pelas quais os estudos a respeito das RPM passaram. Destaca-se, portanto, a importância de futuro estudos que possam superar tais limitações, além da realização de revisões de literatura referentes também ao uso de outros instrumentos psicológicos, para que possam ser conhecidos e analisados os avanços e as limitações no quadro geral de pesquisas envolvendo tais instrumentos.

\section{Referências}

Alves, I. C. B. (1998). Variáveis significativas na avaliação da inteligência. Psicologia Escolar e Educacional, 2(2), 109-114. https://doi.org/10.1590/S1413-85571998000200005

Anache, A. A., \& Corrêa, F. B. (2010). As políticas do Conselho Federal de Psicologia para a avaliação psicológica. In Conselho Federal de Psicologia (Org.), Avaliação psicológica: diretrizes na regulamentação da profissão (pp. 19-30). Brasília, DF: o autor.

Angelini, A. L., Alves, I. C. B., Custódio, E. M., Duarte, W. F., \& Duarte, J. L. M. (1999). Manual matrizes progressivas coloridas de Raven: escala especial. São Paulo, SP: CETEPP.

Bandeira, D. R., Alves, I. C. B., Giacomel, A. E., \& Lorenzatto, L. (2004). Matrizes progressivas coloridas de Raven - Escala Especial: normas para Porto Alegre, RS. Psicologia em Estudo, 9(3), 479-486. https://doi.org/10.1590/S1413-73722004000300016

Bandeira, D. R., Costa, A., \& Arteche, A. (2008). Estudo de validade do DFH como medida de desenvolvimento cognitivo infantil. Psicologia: Reflexão e Crítica, 21(2), 332-337. https://doi.org/10.1590/S0102-79722008000200020

Barbosa, M. C. D. L, Simonetti, L. G., \& Rangel, M. (2005). Relato da vida escolar de pessoas com o transtorno obsessivo-compulsivo e altas habilidades: a necessidade de programas de enriquecimento. Revista Brasileira de Educação Ambiental, 11(2), 201-222. https://doi.org/10.1590/S1413-65382005000200004

Bordin, M. B. M., Linhares, M. B. M., \& Jorge, S. M. (2001). Aspectos cognitivos e comportamentais na média meninice de crianças nascidas pré-termo e com muito baixo peso. Psicologia: Teoria e Pesquisa, 17(1), 49-57. https://doi.org/10.1590/S0102-37722001000100008

Busnello,F.B.,Jou,G.I.,\&Sperb,T.M.(2012).Desenvolvimentodehabilidadesmetacognitivas:capacitaçãodeprofessoresde ensino fundamental. Psicologia: Reflexão e Crítica, 25(2), 311-319. https://doi.org/10.1590/S0102-79722012000200013

Capovilla, F. C., Capovilla, A. G. S., Macedo, E. C., Costa, C. E., \& Duduchi, M. (1996). Manipulação de envolvimento de ego via para-instruções experimentais: efeitos sobre estados de ânimo e desempenho edutivo em resolução de problemas. Psicologia USP, 7(1-2), 143-182. https://doi.org/10.1590/S1678-51771996000100008

Cardoso,A.M.S.,Silva,M.M.,\&Pereira,M.M.B. (2013).Consciênciafonológicaeamemóriadetrabalhodecriançascom e sem dificuldades na alfabetização. CoDAS, 25(2), 110-114. https://doi.org/10.1590/S2317-17822013000200004 
Carvalho, A. E. V.; Linhares, M. B. M.; \& Martinez, F. E. (2001). História de desenvolvimento e comportamento de crianças nascidas pré-termo e baixo peso. Psicologia: Reflexão e Crítica, 14(1), 49-57. https://doi.org/10.1590/S0102-37722001000100008

Conselho Federal de Psicologia. (2011). Ano da avaliação psicológica: textos geradores. Brasília, DF: o autor.

Conselho Federal de Psicologia. (2001). Resolução CFP No 025/2001. Define teste psicológico como método de avaliação privativo do psicólogo e regulamenta sua elaboração, comercialização e uso. Recuperado de http://site.cfp. org.br/wp-content/uploads/2001/11/resolucao2001_25.pdf

Conselho Federal de Psicologia. (2003). Resolução CFP No 002/2003. Define e regulamenta o uso, a elaboração e a comercialização de testes psicológicos e revoga a Resolução CFP nº 025/2001. Recuperado de http://site.cfp.org. br/wp-content/uploads/2003/03/resolucao2003_02_Anexo.pdf

Costa, D. S., Paula, J. J., Alvim-Soares Júnior, A. M., Diniz, B. S., Romano-Silva, M. A., Malloy-Diniz, L. F. et al. (2014). ADHD inattentive symptoms mediate the relationship between intelligence and academic performance in children aged 6-14. Revista Brasileira de Psiquiatria, 36(4), 313-321. https://doi.org/10.1590/1516-4446-2013-1201

Czermainski, F. R., Riesgo, R. S., Guimarães, L. S. P., Salles, J. F., \& Bosa, C. A. (2014). Executive functions in children and adolescents with autism spectrum disorder. Paidéia (Ribeirão Preto), 24(57), 85-94. https:// doi.org/10.1590/1982-43272457201411

DaSilveira, A. C., \& Gomes, W. B. (2012). Perspectiva experiencial da conversa interna no contexto de resolução de problemas. Paidéia (Ribeirão Preto), 22(51), 43-52. https://doi.org/10.1590/S0103-863X2012000100006

Dell'Aglio, D. D., \& Hutz, C. S. (2004). Depressão e desempenho escolar em crianças e adolescentes institucionalizados. Psicologia: Reflexão e Crítica, 17(3), 341-350. https://doi.org/10.1590/S0102-79722004000300008

Dias, T. L., Enumo, S. R. F. (2006). Criatividade e dificuldade de aprendizagem: avaliação com procedimentos tradicional e assistido. Psicologia: Teoria e Pesquisa, 22(1), 69-78. https://doi.org/10.1590/S0102-37722006000100009

Dias, T. L., Enumo, S. R. F., \& Azevedo Junior, R. R. (2004). Influências de um programa de criatividade no desempenho cognitivo e acadêmico de alunos com dificuldade de aprendizagem. Psicologia em Estudo, 9(3), 429-437. https:// doi.org/10.1590/S1413-73722004000300011

Ferreira,A.A.,Conte,K.M.,\&Maturano,E.M. (2011).Meninoscomqueixaescolar:autopercepções, desempenhoecomportamento. EstudosdePsicologia(Campinas),28(4),443-451.https://doi.org/10.1590/S0103-166X2011000400005

Ferreira,V.K.L.,Ferreira,G.V.D.,Lima,J.M.B.,\&Cruz,M.S.(2013).Desempenhointelectualnaexposiçãoalcoólicafetal:relato de série de 10 casos. Jornal Brasileiro de Psiquiatria, 62(3), 234-239. https:// doi.org/10.1590/S0047-20852013000300008

Ferriolli, S. H. T., Linhares, M. B. M., Loureiro, S. R., \& Marturano, E.M. (2001). Indicadores de potencial de aprendizagem obtidos através da avaliação assistida. Psicologia: Reflexão e Crítica, 14(1), 35-43. https://doi.org/10.1590/S0102-79722001000100003

Flores-Mendoza, C., Jardim, G. L., Abad, F. J., \& Rodrigues, L. A. (2010). Informação geral e atual e sua relação com a inteligência e a personalidade em crianças escolares. Estudos de Psicologia (Campinas), 27(2), 161-168. https://doi.org/10.1590/S0103-166X2010000200003

Flores-Mendoza, C. E., Mansur-Alves, M., Abad, F. J., \& Lelé, Á. J. (2010). O que mede o desenho da figura humana?: Estudos de validade convergente e discriminante. Boletim de Psicologia, 60(132), 73-84. Recuperado de http:// pepsic.bvsalud.org/scielo.php?script=sci_arttext\&pid=S0006-59432010000100007\&lng=pt\&tlng=pt

Flores-Mendoza, C. E., Mansur-Alves, M., Lelé, A. J., \& Bandeira, D. R. (2007). Inexistência de diferenças de sexo no fator g (inteligência geral) e nas habilidades específicas em crianças de duas capitais brasileiras. Psicologia: Reflexão e Crítica, 20(3), 499-506. https://doi.org/10.1590/S0102-79722007000300018

Flores-Mendoza, C. E., \& Nascimento, E. (2007). Condição cognitiva de crianças de zona rural. Estudos de Psicologia (Campinas), 24(1), 13-22. https://doi.org/10.1590/S0103-166X2007000100002

Flores-Mendoza, C., Widaman, K. F., Bacelar, T. D., \& Lelé, Á. J. (2014). Propriedades psicométricas do Raven Geral no contexto de Minas Gerais. Arquivos Brasileiros de Psicologia, 66 (2), 1-16. Recuperado de http://seer.psicologia.ufrj.br/index.php/abp/article/view/847/856

Flores-Mendoza, C., Widaman, K. F., Mansur-Alves, M., Silva Filho, J. H., Pasian, S. R., \& Schlottfeldt, C. G. M. F. (2012). Considerations about IQ and human capital in Brazil. Temas em Psicologia, 20(1), 133-154. Recuperado de http://pepsic.bvsalud.org/pdf/tp/v20n1/v20nlall.pdf 
Fonseca, A. L. M., Albernaz, E. P., Kaufmann, C. C., Neves, I. H., \& Figueiredo, V. L. M. (2013). Impacto do aleitamento materno no coeficiente de inteligência de crianças de oito anos de idade. Jornal de Pediatria, 89(4), 346-353. https://doi.org/10.1016/j.jped.2012.12.010

Fonseca, L. C., Tedrus, G. M. A. S., Martins, S. M. V., Gibert, M. A. P., Antunes, T. A., \& Laloni, D. T. (2003). Eletrencefalograma quantitativo em escolares sadios: análise de frequências. São Paulo: Arquivos de Neuro-psiquiatria, 61(3B), 796-801. https://doi.org/10.1590/S0004-282X2003000500018

Fonseca, L. C., Tedrus, G. M. A. S., Pacheco, E. M. C., Berretta, M. F., Campregher, A. A., \& Costa, D. M. (2007). Benign childhood epilepsy with centro-temporal spikes: correlation between clinical, cognitive and EEG aspects. Arquivos de Neuro-psiquiatria, 65(3A), 569-575. https://doi.org/10.1590/S0004-282X2010000400014

Gera, A.A. S.,Linhares, M. B. M. (2006). Avaliação cognitiva assistida: estratégias de perguntas de busca de informaçãona resoluçãodeproblemas.Psicologia:ReflexãoeCrítica, 19(1),74-82.https://doi.org/10.1590/S0102-79722006000100011

Guilhoto, L. M. F., Fernandes, R. D. C., Pachecho, S. P., Ballester, D., \& Gilio, A. E. (2009). Benign focal seizures of adolescence and neuropsychological findings in patients from community. Journal of Epilepsy and Clinical Neurophysiology,15(4), 184-191. https://doi.org/10.1590/S1676-26492009000400009

Ikeda, F. H., Horta, P. A. C., Bruscato, W. L., \& Dolci, J. E. L. (2012). Avaliação do desempenho intelectual e escolar de crianças submetidas à tonsilectomia e adenoamigdalectomia no pré e pós-operatório. Brazilian Journal of Otorhinolaryngology, 78(4), 17-13. https://doi.org/10.1590/S1808-86942012000400005

Joly, M. C. A. R., Berberian, A. A., Andrade R. G., \& Teixeira, T. C. (2010). Análise de teses e dissertações em avaliação psicológica disponíveis na BVS-PSI Brasil. Psicologia: Ciência e Profissão, 30(1), 174-187. https://doi.org/10.1590/S1414-98932010000100013

Joly, M. C. R. A., Silva, M. C. R., Nunes, M. F. O., \& Souza, M. S. (2007). Análise da produção científica em painéis dos congressos brasileiros de avaliação psicológica. Avaliação Psicológica, 6(2), 239-252. Recuperado de http:// pepsic.bvsalud.org/scielo.php?script=sci_arttext\&pid=S1677-04712007000200013

Laros, J. A., Reis, R. F., \& Tellegen, P. J. (2010). Indicações da Validade Convergente do Teste Não-Verbal de Inteligência SON-R 21/2-7[A]. Avaliação Psicológica, 9(1), 43-52. Recuperado de http://pepsic.bvsalud.org/scielo.php?script=sci_arttext\&pid=S1677-04712010000100006

Leon, V., Bosa, C., Hugo, C., \& Hutz, C. S. (2004). Propriedades psicométricas do Perfil Psicoeducacional Revisado: PEP-R. Avaliação Psicológica, 3(1), 39-52. Recuperado de http://pepsic.bvsalud.org/scielo.php?script=sci_abstract\&pid=S1677-04712004000100005

Linhares, M. B. M., Carvalho, A. E. V., Bordin, M. B. M., Chimello, J. T., Martinez, F. E., \& Jorge, S. M. (2000). Prematuridade e muito baixo peso como fatores de risco ao desenvolvimento da criança. Paidéia (Ribeirão Preto), 10(18), 60-69. https:// doi.org/10.1590/S0103-863X2000000100006

Linhares, M. B., Chimello, J. T., Bordin, M. B., Carvalho, A. E., Martinez, C. F. (2005). Desenvolvimento psicológico na fase escolar de crianças nascidas pré-termo em comparação com crianças nascidas a termo. Psicologia: Reflexão e Crítica, 18(1), 109-117. https://doi.org/10.1590/S0102-79722005000100015

Lobato, L., Miranda, A., Faria, I. M., Bethony, J. M., \& Gazzinelli, M. F. (2012). Development of cognitive abilities of children infected with helminths through health education. Revista da Sociedade Brasileira de Medicina Tropical, 45(4), 514-519. https://doi.org/10.1590/S0037-86822012000400020

Lukasova, K., Macedo, E. C., Valois, M. C., Macedo, G. C., \& Schwartzman, J. S. (2007). Percepção de expressões faciais em pessoas com esquizofrenia: movimentos oculares, sintomatologia e nível intelectual. Psico-USF, 12(1), 95-102. https:// doi.org/10.1590/S1413-82712007000100011

Macedo, C. S., Andreucci, L. C., \& Montelli, T. C. B. (2004). Alterações cognitivas em escolares de classe socio-econômica desfavorecida: resultados de intervenção psicopedagógica. Arquivos de Neuro-psiquiatria, 62(3B), 852-857. https://doi.org/10.1590/S0004-282X2004000500021

Maia, R. C. C., Soares, A. B., \& Victoria, M. S. (2009). Um estudo com professores da educação infantil e do ensino fundamental sobre suas habilidades sociais e inteligência geral. Estudos e Pesquisas em Psicologia, 9(2), 464-479.

Mansur-Alves, M., Flores-Mendoza, C., Tierra-Criollo, C. J. (2013). Evidências preliminares da efetividade do treinamento cognitivo para melhorar a inteligência de crianças escolares. Psicologia: Reflexão e Crítica, 26(3), 423-434. https://doi.org/10.1590/S0102-79722013000300001 
Marín Rueda, F. J., \& Sisto, F. F. (2006). Estudo sobre as categorias de interpretação das Matrizes Coloridas de Raven e DFH-Escala Sisto. Aletheia, (23), 17-26. Recuperado de http://pepsic.bvsalud.org/scielo.php?script=sci_arttext\&pid=S1413-03942006000200003

Mian, L., Tango, L. A., Lopes, J., \& Loureiro, S. R. (2009). A depressão materna e o comportamento de crianças em idade escolar. Psicologia: Teoria e Pesquisa, 25(1), 029-037. https://doi.org/10.1590/S0102-37722009000100004

Murphy, C. F. B., Zachi, E. C., Roque, D. T., Ventura, D. S. F., \& Schochat, E. (2014). Influence of memory, attention, IQ and age on auditory temporal processing tests: preliminary study. CoDAS, 26(2), 105-111. https://doi.org/10.1590/2317-1782/2014494IN

Novaes, C. B., Mishima, F, \& Santos, P. L. (2013). Treinamento breve de consciência fonológica: impacto sobre a alfabetização. Revista Psicopedagogia, 30(93), 189-200. Recuperado de http://pepsic.bvsalud.org/scielo.php?script=sci_arttext\&pid=S0103-84862013000300005

Nunes, C. H. S. S., Melzer, A. R. R. M., Rodrigues, G. T., Guisso, L., Sotili, M., Oliveira, C. M. et al. (2012). Evidências de validade do raven MPA pela sua relação com a prova de raciocínio abstrato. Avaliação Psicológica, 11(2), 265-274. Recuperado de http://pepsic.bvsalud.org/scielo.php?pid=S1677-04712012000200012\&script=sci_abstract

Ogusuko, M. T., Lukasova, K., \& Macedo, E. C. (2008). Movimentos oculares na leitura de palavras isoladas por jovens e adultos em alfabetização. Psicologia: Teoria e Prática, 10(1), 113-124. Recuperado de http://pepsic.bvsalud.org/scielo.php?script=sci_arttext\&pid=S1516-36872008000100009

Okano, C. B., Loureiro, S. R., Linhares, M. B. M., \& Maturano, E. M. (2004). Crianças com dificuldades escolares atendidas em programa de suporte psicopedagógico na escola: avaliação do autoconceito. Psicologia: Reflexão e Crítica, 17(1), 121-128. https://doi.org/10.1590/S0102-79722004000100015

Osborn, E., \& Pereira, L. D. (2012). Aspectos neuropsicológicos de crianças de 10 anos de idade. Einstein (São Paulo), 29(2), 433-438. https://doi.org/10.1590/S1679-45082012000400007

Pacanaro, S. V., Alves, G. A. S., Rabelo, I. S., Leme, I. F. A. S., \& Ambiel, R. A. M. (2011). Panorama atual dos testes psicológicos no Brasil de 2003 a 2011. In R. A. M. Ambiel, I. S. Rabelo, S. V. Pacanaro, G. A. S. Alves, \& I. F. A. S. Leme (Orgs.). Avaliação psicológica: guia de consulta para estudantes e profissionais de Psicologia. São Paulo, SP: Casa do Psicólogo.

Pasquali, L. (Org.) (2001). Técnicas de exame psicológico (TEP): manual (Vol. 1: Fundamentos das técnicas psicológicas). São Paulo, SP: Casa do Psicólogo.

Pasquali, L., Wechsler, S., \& Bensusan, E. (2002). Matrizes progressivas do Raven infantil: um estudo de validação para o Brasil. Avaliação Psicológica, 1(2), 95-110. Recuperado de http://pepsic.bvsalud.org/scielo.php?scrip$\mathrm{t}=\mathrm{sci} \_$arttext\&pid=S1677-04712002000200003

Paula, K. M. P., \& Enumo, S. R. F. (2007). Avaliação assistida e comunicação alternativa: procedimentos para a educação inclusiva. Revista Brasileira de Educação Especial, 13(1), 3-26. https://doi.org/10.1590/S1413-65382007000100002

Paulino, J. A., \& Lopes, R. F. F. (2010). Relação entre percepção e comportamento de risco e níveis de habilidades cognitivas em um grupo de adolescentes em situação de vulnerabilidade social. Psicologia: Ciência e Profissão, 30(4), 752-765. https://doi.org/10.1590/S1414-98932010000400007

Pawlowski, J., Fonseca, R. P., Salles, J. F., Parente, M. A. M. P., \& Bandeira, D. R. (2008). Evidências de validade do Instrumento de Avaliação Neuropsicológica Breve Neupsilin. Arquivos Brasileiros de Psicologia, 60(2), 101-116. Recuperado de http://pepsic.bvsalud.org/scielo.php?script=sci_arttext\&pid=S1809-52672008000200011

Pereira, P.C., Santos, A. B., \&Williams, L. C. A. (2009). Desempenho escolar da criança vitimizada encaminhada ao fórum judicial. Brasília: Psicologia: Teoria e Pesquisa, 25(1), 19-28. https://doi.org/10.1590/S0102-37722009000100003

Primi, R. (2010). Avaliação psicológica no Brasil: fundamentos, situação atual e direções para o futuro. Psicologia: Teoria e Pesquisa, 26(n. spe), 25-35. https://doi.org/10.1590/S0102-37722010000500003

Queiroz, O. A., Enumo, S. R. F., \& Primi, R. (2013). Desempenho de crianças com e sem necessidades especiais em provas assistidas e psicométricas. Revista Brasileira de Educação Especial, 19(3), 425-446. https://doi.org/10.1590/S1413-65382013000300009

Ribeiro,R.K.S.M.,Semer,N.L.,\&Yazigi,L. (2011).RorschachComprehensiveSystemnormsinBrazilianchildrenfrompublic and private schools. Psicologia: Reflexão e Crítica, 24(4), 671-684. https://doi.org/10.1590/S0102-79722011000400007 
Rodrigues, V. S., Caminha, R. M., \& Horta, R. L. (2006). Déficits cognitivos em pacientes usuários de crack. Revista Brasileira de Terapias Cognitivas, 2(1), 67-72. Recuperado de http://pepsic.bvsalud.org/scielo.php?script=sci_ arttext\&pid=S1808-56872006000100007

Rosseti, M. O., Rabelo, I. S., Leme, I. F. A. S., Pacanaro, S. V., \& Güntert, I. B. (2009). Evidências de validade das Matrizes Progressivas Avançadas de Raven em universitários. Psico-USF, 14(2), 177-184. https://doi.org/10.1590/S1413-82712009000200006

Santos, D. N., Borges A. P. V., Pereira, P. S., Chalhub, A. A., Happé, F., Silva, R. C. R. et al. (2002). Epidemiologia do desenvolvimento cognitivo de escolares em Jequié, Bahia, Brasil: procedimentos de avaliação e resultados gerais. Caderno de Saúde Pública, 18(3), 723-733. https://doi.org/10.1590/S0102-311X2002000300021

Santos, M. A, Raspantini, R. L., Silva, L. A. M., \& Escrivão, M. V. (2003). Dos laços de sangue aos laços de ternura: o processo de construção da parentalidade nos pais adotivos. Psic: Revista da Vetor Editora, 4(1), 14-21. Recuperado de http://pepsic.bvsalud.org/scielo.php?script=sci_arttext\&pid=S1676-73142003000100003

Santos, M. T., \& Nascimento, E. (2012). Inteligência e personalidade: um estudo correlacional em uma amostra de universitários. Estudos de Psicologia (Campinas), 29(2), 163-171. https://doi.org/10.1590/S0103-166X2012000200002

Saur, A. M., Correia, S. K. B., Bettiol, H., Barbieri, M. A., \& Loureiro, S. R. (2014). Variables associated with cognitive, behavioral and emotional development: a cohort of schoolchildren. Psico-USF, 19(1), 131-141. https://doi.org/10.1590/S1413-82712014000100013

Silva, G. B. A., Zaninotto, A. L. C., Lucia, M. C. S., \& Scaff, M. (2012). Avaliação do desempenho da memória de curto prazo em crianças de escola pública e particular. Psicologia Hospitalar, 10(1), 80-94. Recuperado de http://pepsic.bvsalud.org/scielo.php?script=sci_arttext\&pid=S1677-74092012000100006

Silva, P. A., \& Santos, F. H. (2011). Discalculia do desenvolvimento: avaliação da representação numérica pela ZAREKI-R. Psicologia: Teoria e Pesquisa, 27(2), 169-177. https://doi.org/10.1590/S0102-37722011000200003

Silva, R. C. R., Assis, A. M. O., Hasselmann, M. H., Santos, L. M., Pinto, E. J., \& Rodrigues, L. C. (2012). Influência da violência familiar na associação entre desnutrição e baixo desenvolvimento cognitivo. Jornal de Pediatria, 88(2), 149-154. https://doi.org/10.2223/JPED.2176

Sisto, F. F., \& Marin Rueda, F. J., \& Bartholomeu, D. (2006). Estudo sobre a unidimensionalidade do teste Matrizes Progressivas Coloridas de Raven. Psicologia: Reflexão e Critica, 9(1), 66-73. https://doi.org/10.1590/S0102-79722006000100010

Soares, A. B., Francischetto, V., Peçanha, A. P. C. L., Miranda, J. M., \& Dutra, B. M. S. (2013). Intelligence and social competence in university adaptation. Estudos de Psicologia (Campinas), 30(3), 317-328. https://doi.org/10.1590/S0103-166X2013000300001

Souza, C. C., \& Resende, A. C. (2012). Transtornos psicológicos em adolescentes socioeducandos. Avaliação Psicológica, 11(1), 95-109. Recuperado de http://pepsic.bvsalud.org/scielo.php?script=sci_arttext\&pi$\mathrm{d}=\mathrm{S} 1677-04712012000100010$

Souza Filho, M. L., Belo, R., \& Gouveia,V.V. (2006) Testes psicológicos: análise da produção científica brasileira no período 2000-2004. Psicologia:Ciência e Profissão, 26(3), 478-489. https://doi.org/10.1590/S1414-98932006000300011

Urbina, S. (2007). Fundamentos da testagem psicológica. Porto Alegre, RS: Artmed.

Varanda, C. A., \& Fernandes, F. D. M. (2011). Consciência sintática: prováveis correlações com a coerência central e a inteligência não-verbal no autismo. Jornal da Sociedade Brasileira de Fonoaudiologia, 23 (2), 142-51. https://doi.org/10.1590/S2179-64912011000200011

Werlang, B. S. G.; Nunes, C. H. S. S., \& Borges, V. R. (2014). Evidências de validade com base na estrutura interna no Teste dos Contos de Fadas. Psico-USF, 19(1), 107-118. https://doi.org/10.1590/S1413-82712014000100011

Wigg, C. M. D., \& Duro, L. A. A. (1995). Estudo psicológico longitudinal na distrofia miotônica. Arquivos de Neuro-psiquiatria, 53(4), 749-754. https://doi.org/10.1590/S0004-282X1995000500006

Witter, G. P. (1999). Metaciência e leitura. In G. P. Witter (Org.), Leitura: textos e pesquisas (pp. 13-22). Campinas, SP: Alínea.

Zampieri, M., Shelini, P. W., \& Crespo, C. R. (2012). Eficácia de um programa de estimulação de capacidades intelectuais. Estudos de Psicologia (Campinas), 29(3), 353-362. https://doi.org/10.1590/S0103-166X2012000300005 


\section{Lucila Moraes Cardoso}

Doutora em Psicologia pela Universidade São Francisco, São Paulo - SP. Docente da Universidade Estadual do Ceará, Fortaleza - CE. Brasil.

E-mail: lucilamcardoso@yahoo.com.br

Érica Ive Xavier Lopes

Especialista em Saúde Mental pela Universidade Estadual do Ceará, Fortaleza - CE. Brasil.

E-mail: ericaive@hotmail.com

Jamille Cavalcante de Oliveira

Residente de Psicologia, ênfase em Saúde Mental, no Hospital Universitário Walter Cantídio (HUWC - UFC), Fortaleza-CE. Brasil.

E-mail: milly_edh@hotmail.com

\section{Aline Pinheiro Braga}

Especialização em Saúde Pública em andamento pela Universidade Estadual do Ceará, Fortaleza - CE. Brasil. E-mail: liinebraga@gmail.com

Endereço para envio de correspondência:

A/c Prof. Dra. Lucila Moraes Cardoso

Curso de Psicologia - Universidade Estadual do Ceará (UECE)

Av. Dr. Silas Munguba, 1700, Campus do Itaperi, Fortaleza-CE

CEP: 60.714 .903

Recebido 22/01/2015

Aprovado 14/12/2016

Received $01 / 22 / 2015$

Approved 12/14/2016

Recibido 22/01/2015

Aceptado 14/12/2016

Como citar: Cardoso, L. M., Lopes, E. I. X., Oliveira, J. C., \& Braga, A. P. (2017). Análise da produção científica brasileira sobre o Teste das Matrizes Progressivas de Raven. Psicologia: Ciência e Profissão, 37(1): 62-77. https://doi.org/10.1590/1982-3703000212015

How to cite: Cardoso, L. M., Lopes, E. I. X., Oliveira, J. C., \& Braga, A. P. (2017). Analysis of the Brazilian scientific production on the Raven's Progressive Matrices Test. Psicologia: Ciência e Profissão, 37(1): 62-77. https://doi.org/10.1590/1982-3703000212015

Cómo citar: Cardoso, L. M., Lopes, E. I. X., Oliveira, J. C., \& Braga, A. P. (2017). Análisis de la producción científica brasileña acerca de la Prueba de Raven. Psicologia: Ciência e Profissão, 37(1): 62-77.

https://doi.org/10.1590/1982-3703000212015 